\title{
Gribov horizon under the (lattice) microscope ${ }^{\dagger}$
}

\section{Jeff Greensite}

The Niels Bohr Institute, Blegdamsvej 17, DK-2100 Copenhagen Ø, Denmark;

Physics and Astronomy Dept., San Francisco State University, San Francisco, CA 94117, USA

E-mail: greensitestars.sfsu.edu

\section{Štefan Olejník*}

Institute of Physics, Slovak Academy of Sciences, SK-845 11 Bratislava, Slovakia

E-mail: stefan.olejnikesavba.sk

\section{Daniel Zwanziger}

Physics Department, New York University, New York, NY 10003, USA

E-mail: daniel.zwanziger@nyu.edu

The infinite color-Coulomb energy of color-charged states is related to enhanced density of nearzero modes of the Faddeev-Popov operator in Coulomb gauge. We confirm the enhancement in numerical simulations and show that it is tied to the presence of percolating center vortex configurations.

XXIIIrd International Symposium on Lattice Field Theory

25-30 July 2005

Trinity College, Dublin, Ireland

\footnotetext{
$\dagger$ Our research is supported in part by the U.S. Department of Energy under Grant No. DE-FG03-92ER40711 (J.G.), the Slovak Science and Technology Assistance Agency, Grant No. APVT-51-005704 (Š.O.), and the National Science Foundation, Grant No. PHY-0099393 (D.Z.).

*Speaker.
} 


\section{Introduction: Confinement scenario in Coulomb gauge}

The Faddeev-Popov operator in Coulomb gauge

$$
M(A) \equiv-\nabla \cdot \mathscr{D}(A), \quad \text { where } \quad \mathscr{D}_{i}^{a c}(A)=\partial_{i} \delta^{a c}+f^{a b c} A_{i}^{b}(x),
$$

plays a crucial role in the Gribov-Zwanziger confinement scenario [1], 2]. It enters the kernel $K$ in the (classical) Coulomb energy of a color charge distribution $\rho$ :

$$
H_{\text {coul }}=\frac{1}{2} \int d^{3} \mathbf{x} d^{3} \mathbf{y} \rho^{a}(x) K^{a b}(x, y ; A) \rho^{b}(y), \quad \rho^{a}=\rho_{\text {matter }}^{a}-f^{a b c} \mathbf{A}^{b} \cdot \mathbf{E}^{c},
$$

where

$$
K^{a b}(x, y ; A) \equiv\left[M^{-1}\left(-\nabla^{2}\right) M^{-1}\right]_{x, y}^{a, b} .
$$

The essence of the scenario can be summarized in the following way: The Coulomb-gauge condition $\nabla \cdot \mathbf{A}^{a}=0$ does not fix the gauge completely. Gribov [1] suggested to restrict to the subspace of transverse gauge fields for which the Faddeev-Popov operator is positive, i.e. local minima with respect to $g(x)$ of

$$
I[\mathbf{A}, g]=\int d x\left[{ }^{g} \mathbf{A}^{a}(x)\right]^{2}, \quad \text { where }{ }^{g} A_{i}=g^{-1} A_{i} g+g^{-1} \partial_{i} g .
$$

The boundary of this Gribov region (GR) is called the Gribov horizon. However, even this does not eliminate the Coulomb-gauge ambiguities completely, one has to further narrow the gauge-field configuration space to the fundamental modular region (FMR), i.e. the set of absolute minima of the functional (1.4). Both the GR and the FMR are bounded in every direction and convex. The dimension of the gauge-field configuration space is huge, so it is reasonable to expect that most configurations are located close to its boundary (horizon). The interaction kernel $K$ contains the inverse of the FP operator, which is strictly zero on the horizon and near-zero close to the horizon. A high density of configurations near the horizon leads to a strong enhancement of the Coulomb interaction energy, and hopefully causes color confinement.

In this contribution we formulate a simple criterion of confinement for static color charges through properties of eigenstates of the FP operator in Coulomb gauge close to the Gribov horizon, and then discuss how the fulfillment of this criterion depends on presence/absence of center vortices. Details, as well as some analytic insights on the connections between center vortices and the Gribov horizon, can be found in a recent publication [3]. ${ }^{1}$

\section{Lattice Faddeev-Popov operator and its eigenstates}

If we parametrize link variables in $\mathrm{SU}(2)$ lattice gauge theory by

$$
U_{\mu}(x)=b_{\mu}(x)+i \sigma^{c} a_{\mu}^{c}(x), \quad b_{\mu}(x)^{2}+\sum_{c} a_{\mu}^{c}(x)^{2}=1,
$$

\footnotetext{
${ }^{1}$ We have also investigated localization properties of the lowest nontrivial eigenvectors of the Faddeev-Popov operator in Coulomb gauge. These were discussed in Jeff Greensite's talk at this conference and in Sect. V of Ref. [4].
} 
the lattice Faddeev-Popov operator in Coulomb gauge is given by the following expression:

$$
\begin{aligned}
M_{x y}^{a b} & =\delta^{a b} \sum_{k}\left\{\delta_{x y}\left[b_{k}(x)+b_{k}(x-\hat{k})\right]-\delta_{x, y-\hat{k}} b_{k}(x)-\delta_{y, x-\hat{k}} b_{k}(y)\right\} \\
& -\varepsilon^{a b c} \sum_{k}\left\{\delta_{x, y-\hat{k}} a_{k}^{c}(x)-\delta_{y, x-\hat{k}} a_{k}^{c}(y)\right\} .
\end{aligned}
$$

We are interested in its eigenstates

$$
\sum_{b, y} M_{x y}^{a b} \phi_{y}^{(n) b}=\lambda_{n} \phi_{x}^{(n) a}
$$

in particular in their properties near to the Gribov horizon (i.e. in the limit $\lambda \rightarrow 0$ ). The most relevant quantities are

- the density of eigenstates $\rho(\lambda)$, and

- the average Laplacian:

$$
F_{n} \equiv \sum_{a, x y} \phi_{x}^{(n) a}\left(-\nabla^{2}\right)_{x y} \phi_{y}^{(n) a *}
$$

\section{A confinement condition}

We shall now formulate a simple confinement criterion in terms of properties of eigenstates of the FP operator. The energy of a static color charge state $\Psi_{C}^{\alpha}[A ; x]$ in Coulomb gauge

$$
\mathscr{E}=\frac{\left\langle\Psi_{C}^{\alpha}\left|H_{\text {coul }}\right| \Psi_{C}^{\alpha}\right\rangle}{\left\langle\Psi_{C}^{\alpha} \mid \Psi_{C}^{\alpha}\right\rangle}-\left\langle\Psi_{0}\left|H_{\text {coul }}\right| \Psi_{0}\right\rangle \sim\left\langle K^{a a}(x, x ; A)\right\rangle
$$

can be easily shown to be given by

$$
\mathscr{E}=\frac{1}{3 V_{3}} \sum_{n}\left\langle\frac{F_{n}}{\lambda_{n}^{2}}\right\rangle \quad \text { going to } \quad \int_{0}^{\lambda_{\max }} \frac{d \lambda}{\lambda^{2}}\langle\rho(\lambda) F(\lambda)\rangle \quad \text { for } V_{3} \rightarrow \infty .
$$

An immediate consequence is that the excitation energy $\mathscr{E}$ of a static, unscreened color charge is divergent if, at infinite volume,

$$
\lim _{\lambda \rightarrow 0} \frac{\langle\rho(\lambda) F(\lambda)\rangle}{\lambda}>0 .
$$

This criterion is a necessary but not sufficient condition for confinement; an explicit example will be given at the end of Sect. 5. (It is obviously not fulfilled in the free theory, where $\rho(\lambda) \sim \sqrt{\lambda}$, $F(\lambda)=\lambda$, and consequently $\mathscr{E} \sim \sqrt{\lambda_{\max }}$.)

\section{Three ensembles of lattice configurations}

We will investigate fulfillment of the condition (3.3) in three ensembles of configurations:

1. full configurations, $\left\{U_{\mu}(x)\right\}$;

2. "vortex-only" configurations: these are obtained from full configurations fixed to the (direct) maximal center gauge [5] by center projection, $\left\{Z_{\mu}(x)=\operatorname{sign} \operatorname{Tr}\left[U_{\mu}^{(M C G)}(x)\right]\right\}$;

3. "vortex-removed" configurations, obtained by the recipe of de Forcrand and D'Elia [6]: $\left\{U_{\mu}^{(R)}(x)=Z_{\mu}^{\dagger}(x) U_{\mu}(x)\right\}$.

Each configuration in these three ensembles was brought to Coulomb gauge by maximizing with respect to gauge transformations, on each time slice $\mathscr{R}_{\text {coul }}(t)=\sum_{\mathbf{x}} \sum_{k=1}^{3} \frac{1}{2} \operatorname{Tr}\left[U_{k}(\mathbf{x}, t)\right]$. 

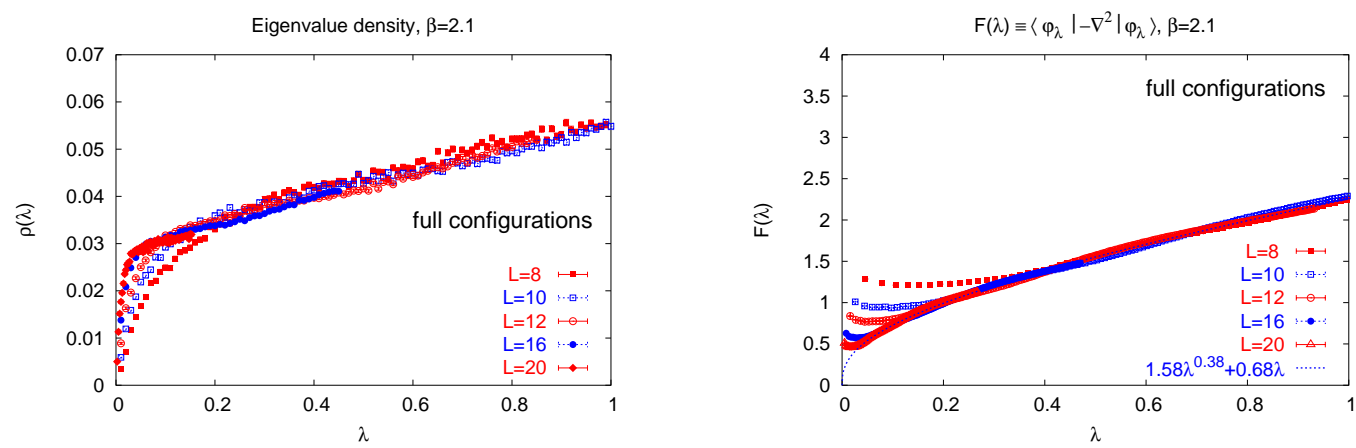

Figure 1: $\rho(\lambda)$ and $F(\lambda)$ for full lattice configurations.

\section{Results}

Pure gauge theory at zero temperature. The results for full configurations at $\beta=2.1$ are shown in Figure 1, for a series of lattice volumes. ${ }^{2}$ Both $\rho(\lambda)$ and $F(\lambda)$ exhibit a sharp "bend" near $\lambda \rightarrow 0$, and behave near 0 like a small power of $\lambda$. A scaling analysis similar to that used in random matrix theory gives the estimates

$$
\rho(\lambda) \sim \lambda^{0.25}, \quad F(\lambda) \sim \lambda^{0.38} .
$$

The confinement condition (3.3) is obviously satisfied, which is a direct manifestation of the mechanism proposed by Gribov and Zwanziger.

The situation in vortex-only configurations is displayed in Figure 国. The enhancement of the density of states is even more pronounced than in full configurations, and both quantities of interest seem to converge to a non-zero value in the infinite volume limit

$$
\rho(0) \sim 0.06, \quad F(0) \sim 1.0 .
$$

(though their proportionality to very small powers of $\lambda$ cannot be excluded). Once again, the condition (3.3) is fulfilled.
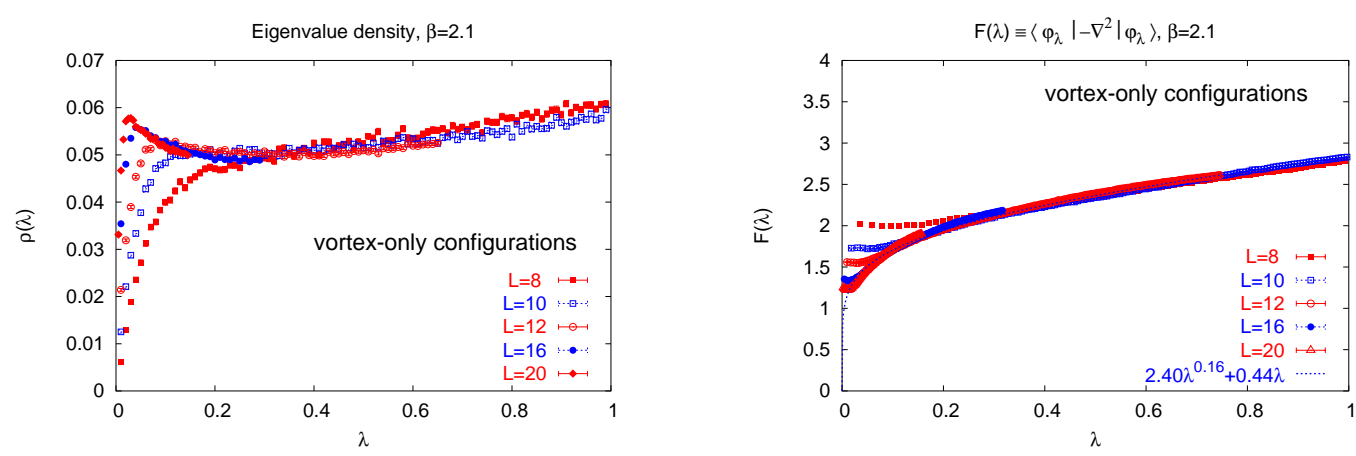

Figure 2: $\rho(\lambda)$ and $F(\lambda)$ for vortex-only (center-projected) configurations.

\footnotetext{
${ }^{2}$ The results for $\beta=2.3$ and 2.4 can be found in Ref. [ [
} 

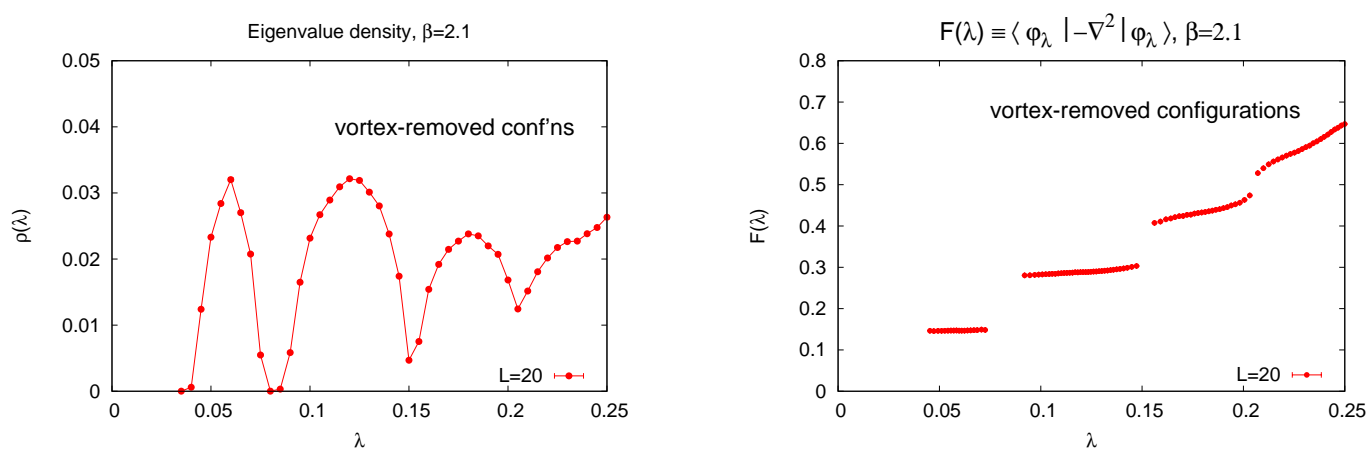

Figure 3: $\rho(\lambda)$ and $F(\lambda)$ for vortex-removed configurations.

The eigenvalue spectrum of the FP operator is drastically different for vortex-removed configurations, see Figure 3 for the largest available, $20^{4}$ lattice. The density displays a series of peaks, and values of $F(\lambda)$ are organized into bands, separated by gaps. This can be understood rather simply: For the Laplacian operator (equal to the FP operator at zero-th order in the gauge coupling) the eigenvalue density, at finite volume, is a sum of delta-functions, and each eigenvalue is multiply degenerate. The vortex-removed configuration seems to be just a small perturbation around the zero-coupling limit, which lifts the degeneracy. In this way, delta-functions in the density of states turn into distinct peaks of finite width, and degenerate values of $F(\lambda)$ spread into bands. The number of values inside the $k$-th band of $F(\lambda)$ exactly matches the degeneracy of the $k$-th eigenvalue of the unperturbed Laplacian operator.

This result demonstrates a deep relation between the Gribov-horizon and center-vortex confinement mechanism. Center vortices seem to be the field configurations providing the mechanism needed for enhancement of eigenvalues near the horizon.

Pure gauge theory in the deconfined phase. A seemingly paradoxical result is obtained above the deconfinement transition: our quantities above $T_{c}$ look the same as at $T=0$ ( $c f$. Figure $\emptyset$ with Figs. 1 and 2)! However, one should keep in mind that spacelike links are a confining ensemble even in the deconfined phase, and spacelike Wilson loops have an area law behavior.

The result for the deconfined phase can be naturally explained in the Gribov-horizon scenario.
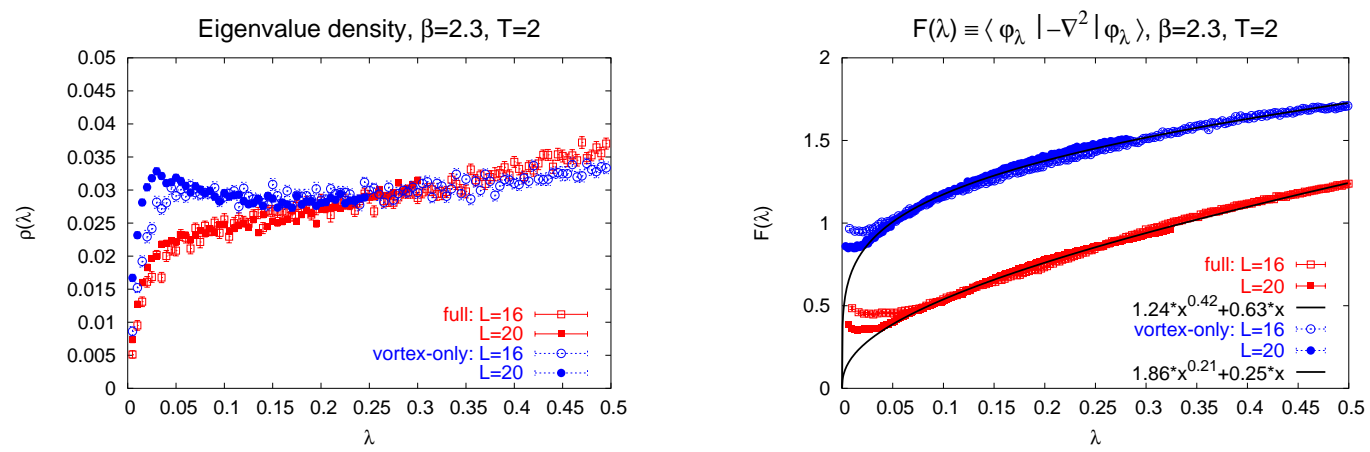

Figure 4: $\rho(\lambda)$ and $F(\lambda)$ in the deconfined phase, for full and vortex-removed configurations. 
In Coulomb gauge the gauge fixing is done independently on each $3 d$ time slice. According to the horizon scenario, on each time slice, $3 d$ configurations $\mathbf{A}(\mathbf{x})$ are favored that lie near the horizon of a $3 d$ gauge theory, and this enhances the instantaneous color-Coulomb potential. This is true for every temperature $T$, including in the deconfined phase, because temperature determines the extent of the lattice in the fourth dimension. Thus, the horizon scenario provides a framework in which confinement may be understood, but it is not detailed enough to tell us under what conditions the infinite color-Coulomb potential may be screened to give a finite self-energy. ${ }^{3}$

\section{Conclusions}

The low-lying eigenvalues of the FP operator in Coulomb gauge tend towards zero as the lattice volume increases. The density of the eigenvalues goes as a small power of $\lambda$, and this, together with a similar behavior of the average Laplacian, $F(\lambda)$, assures the infrared divergence of the energy of an unscreened color charge. These facts support the ideas of the Gribov-horizon confinement scenario.

The constant density of low-lying eigenvalues can be attributed to the vortex component of gauge-field configurations. A thermalized configuration in a pure gauge theory factors into a confining piece (the vortex-only part), and a piece which closely resembles the lattice of a gauge-Higgs theory in the Higgs phase (the vortex-removed configuration). This establishes firm connection between the center-vortex picture and the Gribov-horizon scenario.

The Gribov-Zwanziger scenario, though invented to explain confinement, is operative also in the finite temperature deconfined phase.

Here we only covered results of our numerical investigations. Related analytical developments were omitted and can be found in our recent publication [3].

\section{References}

[1] V. N. Gribov, Quantization of non-Abelian gauge theories, Nucl. Phys. B139 (1978) 1.

[2] D. Zwanziger, Renormalization in the Coulomb gauge and order parameter for confinement in QCD, Nucl. Phys. B518 (1998) 237.

[3] J. Greensite, Š. Olejník, and D. Zwanziger, Center vortices and the Gribov horizon, JHEP 05 (2005) 070 [hep-lat/0407032].

[4] J. Greensite, Š. Olejník, M. Polikarpov, S. Syritsyn, and V. Zakharov, Localized eigenmodes of covariant Laplacians in the Yang-Mills vacuum, Phys. Rev. D 71 (2005) 114507 [hep-lat/ 0504008 ]; Localized eigenmodes of the covariant lattice Laplacian, in proceedings of Lattice 2005, PoS(LAT2005)325.

[5] L. Del Debbio, M. Faber, J. Giedt, J. Greensite, and Š. Olejník, Detection of center vortices in the lattice Yang-Mills vacuum, Phys. Rev. D 58 (1998) 094501 [hep-lat/9801027].

[6] Ph. de Forcrand and M. D'Elia, Relevance of center vortices to QCD, Phys. Rev. Lett. 82 (1999) 4582 [hep-lat/9901020].

[7] D. Zwanziger, Equation of state of gluon plasma from fundamental modular region, Phys. Rev. Lett. 94 (2005) 182301 [hep-ph / 0407103 ]; also in proceedings of Lattice 2005, PoS(LAT2005)191.

\footnotetext{
${ }^{3}$ For another example of operation of the horizon scenario in the deconfined phase see Ref. [7].
} 